\title{
Primary anorectal malignant melanoma treated with neoadjuvant chemoradiotherapy and sphincter-sparing surgery: A case report
}

\author{
MENG SU, LUCHENG ZHU, WENHUA LUO, HANGPING WEI and CHANGLIN ZOU \\ Department of Radio-Chemotherapy Oncology, The First Affiliated Hospital of Wenzhou Medical University, \\ Wenzhou, Zhejiang 325000, P.R. China
}

Received July 30, 2013; Accepted February 13, 2014

DOI: $10.3892 / \mathrm{ol} .2014 .1925$

\begin{abstract}
Primary anorectal (PA) malignant melanoma (MM) is a rare disease associated with a high mortality rate. The most appropriate treatment strategy for PAMM remains controversial. A 55-year-old female patient, who was misdiagnosed with locally advanced rectal carcinoma, was treated with preoperative radiotherapy and concurrent oral capecitabine. During the therapy, grade 1 leukopenia occurred, however, there was no interruption to treatment. Following chemoradiotherapy, a computer tomography scan identified that the tumor had shrunk significantly and the original enlarged lymph nodes had disappeared. Eight weeks after completion of chemoradiotherapy, sphincter-sparing surgery was performed on the patient and based on the postoperative pathological result, MM was diagnosed. At the time of writing, the patient has survived disease-free for 15 months and at the most recent follow-up examination the Karnofsky Performance Scale score was 100 . The therapeutic regimen of neoadjuvant concurrent chemoradiotherapy together with sphincter-sparing surgery is considered to be an optimal choice for patients with PAMM. However, further studies are required to evaluate the efficacy and clinical utility of this therapeutic regimen.
\end{abstract}

\section{Introduction}

Malignant melanoma (MM) arising from the anorectal region is rare, comprising only $0.4-1.6 \%$ of all melanomas and $\sim 1 \%$ of all anal canal tumors $(1,2)$. However, the anorectum is the third most common site for the occurrence of primary MM, preceded only by the skin and eyes (3). Occasionally, primary anorectal (PA) MM is misdiagnosed as anorectal cancer, which is attributed to a lack of immunohistochemical findings. As a

Correspondence to: Professor Changlin Zou, Department of Radio-Chemotherapy Oncology, The First Affiliated Hospital of Wenzhou Medical University, 2 Fuxue Street, Wenzhou, Zhejiang 325000, P.R. China

E-mail: zcl19670115@163.com

Key words: primary anorectal malignant melanoma, neoadjuvant chemoradiotherapy, sphincter-sparing surgery result, there is no consensus on the most effective therapeutic regimen for PAMM. Preoperative concurrent chemoradiotherapy (CCRT) has been widely used for the treatment of numerous malignant neoplastic diseases, however, not in PAMM. The present case report proposes a novel therapeutic regimen for PAMM by demonstrating a patient who received preoperative CCRT and sphincter-sparing surgery. Patient provided written informed consent.

\section{Case report}

A 55-year-old female patient experienced intermittent hematochezia for one year. Digital rectal examination revealed a hard cauliflower-shaped mass (size, 3.0x4.0 cm), located $1 \mathrm{~cm}$ from the anal verge, with bloodstains observed on the finger-cot. The serum levels were as follows: Carcinoembryonic antigen, $2.6 \mu \mathrm{g} / 1$; carbohydrate antigen 19-9, $25.6 \mathrm{U} / \mathrm{ml}$; and lactate dehydrogenase, $180 \mathrm{U} / 1$, which were all considered to be within the normal ranges. Abdominal magnetic resonance imaging (MRI) demonstrated a rectal mass (diameter, $4.2 \mathrm{~cm}$ ), which had invaded the anus and cervix, in addition, the lymph nodes of the right pelvic cavity and right inguen were enlarged. Through colonoscopy, a pigmented mass with a large ulcer was observed on the anterior wall of the anorectum, $\sim 4.0 \mathrm{~cm}$ in size (Fig. 1). The result of colonoscopy pathology indicated low differentiated carcinoma, however, immunohistochemical analysis was not performed. According to the abovementioned findings, the preoperative diagnosis was a locally advanced rectal carcinoma. In order to preserve sphincter function, obtain pathological downstaging and reduce the rate of recurrence, preoperative CCRT was adopted.

A total irradiation dose of $45.0 \mathrm{~Gy}$ was delivered in daily fractions of $1.8 \mathrm{~Gy}$, five times per week, through a pair of opposed anterior-posterior fields using a 6-MV linear accelerator. The treatment fields were set as follows; the superior border was placed at $\mathrm{S} 1$, the inferior border was placed below the anus $(\sim 0.5 \mathrm{~cm})$ and the lateral borders of the planning target volume were $1.5-\mathrm{cm}$ lateral to the widest bony margin of the true pelvic wall. Throughout the radiation period, capecitabine was administered every $12 \mathrm{~h}$, twice a day following a meal, at a dose of $1,000 \mathrm{mg}$ for two weeks. Administration of the medication subsequently ceased for one week. Following this, the medication regimen was continued for another two weeks. 


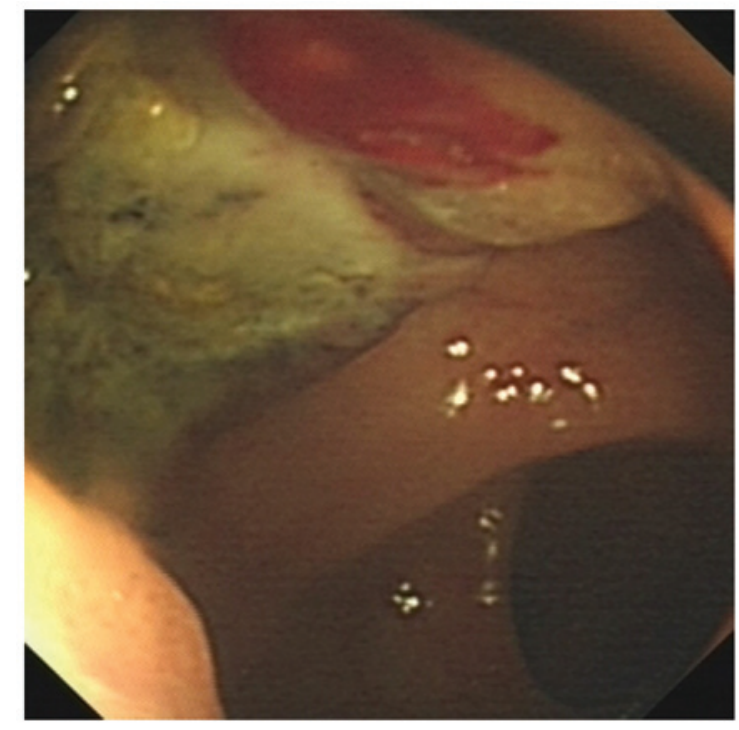

Figure 1. Colonoscopy examination prior to chemoradiotherapy.

During the therapy, only grade 1 leukopenia occurred and the treatment was well-tolerated by the patient, with no regimen interruption.

A digital rectal examination, following CCRT, revealed a hard cauliflower-shaped mass (size, $2.0 \times 2.0 \mathrm{~cm}$ ) without any bloodstaining observed on the finger-cot. The computer tomography scan demonstrated that the tumor had shrunk sharply (diameter, $2.0 \mathrm{~cm}$ ) and there were no enlarged lymph nodes.

The surgery was performed eight weeks following the completion of CCRT. A cauliflower-shaped tumor (size, 2.0x2.0 cm), which invaded the posterior wall of the vagina, was observed on the antetheca of the anal region. However, the enlarged lymph nodes of the right pelvic cavity and right inguen, which were observed in the prior MRI scan, were no longer detected. The rectum, uterus, bilateral accessory and posterior wall of the vagina were excised during surgery. Finally, coloanal anastomosis and reconstruction of the vagina were performed. A histopathological examination demonstrated cells, which exhibited marked cytological atypia, pleomorphism and increased mitotic activity. Numerous melanin granules were apparent between the tumor cells (Fig. 2). Immunohistochemical staining for HMB-45 (weakly positive), Melan-A (positive), S-100 (weakly positive) and VIM (weakly positive) confirmed the diagnosis of PAMM. According to the American Joint Committee on Cancer classification for melanoma (7th edition) (4), the postoperative stage was identified as ypT2aN0M0, stage IB, while the initial stage was cT4bN2M0, stage III. In addition, the resection margins were negative. There was no complication following the surgery and the postoperative recovery was good. The patient did not receive any subsequent adjuvant therapy.

The patient underwent a close postoperative follow-up for 15 months, without any evidence of local relapse, lymphatic metastasis or distant metastasis. As a result of this treatment strategy, the patient obtained complete remission with a high quality of life. The only symptoms experienced by the patient were pain from the incision for one month and occasional instances of constipation in the six months following surgery.

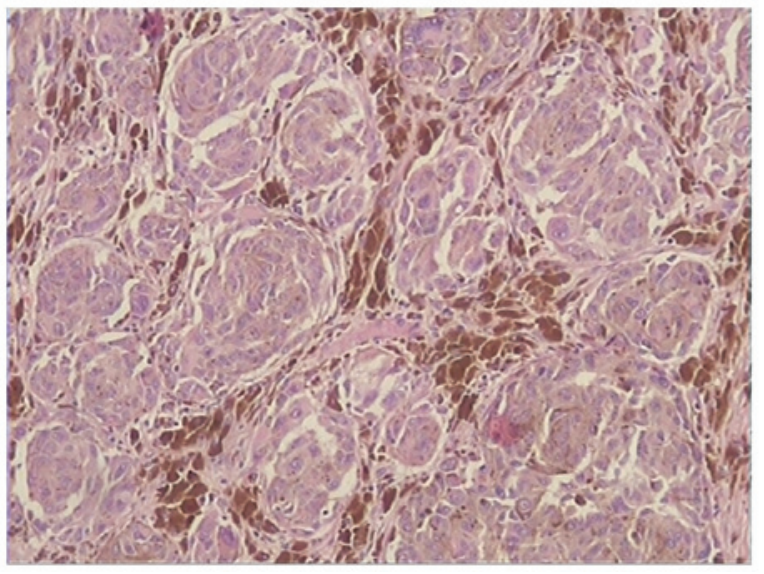

Figure 2. Histopathological examination demonstrated numerous melanin granules between the tumor cells (hematoxylin and eosin staining; magnification, $\mathrm{x} 400)$.

Recently, the patient has not complained of any symptoms or signs. In the postoperative follow-up period, the mean Karnofsky Performance Scale (KPS) score was 90 and the most recent score was 100 .

\section{Discussion}

MM is a neoplasm of neuroectodermal origin arising from melanocytes. PAMM most commonly originates near the anorectal junction, where melanocytes normally occur. Goldman et al (5) reported 49 cases of PAMM, and 45 of those were located at, or near, the anorectal junction. As MM arising from the anorectum was initially described by Moore in 1857 (6), a series of articles describing PAMM have been published. The incidence remains extremely low, ranging between $<1$ and $3 \%$ (7). In addition, PAMM is a malignant disease with an exceptionally poor prognosis. The five-year survival rate is only $9-16 \%(8,9)$, which may be explained by the aggressive nature of the disease. A previous study identified that $26 \%$ of patients had exhibited distant metastasis at the time of diagnosis (10).

PAMM can easily be mistaken for certain other lesions, including hemorrhoids, polyp and adenocarcinoma, and misdiagnosis rates have been recorded as 58.2-86.4\% $(9,11,12)$. There are several common causes for misdiagnosis in PAMM, including i) the symptoms and signs of PAMM are not specific; ii) non-pigmented lesions appear on endoscopic examination; iii) immunohistochemical examination as a definite diagnosis approach is not performed in every colonoscopy pathology; and iv) MM located on the anorectum is rare, and inexperienced physicians lack the vigilance and awareness of this disease.

In the present case, a pigmented mass was revealed on the colonoscopy, however, the main cause of misdiagnosis was that an immunohistochemical examination was not performed. Misdiagnosis of PAMM may lead to a delay in treatment and distant metastasis. In one study, the five-year survival rate of misdiagnosed patients was $11 \%$, compared with $25 \%$ that had been diagnosed correctly (9).

As there is a low incidence of, and inexperience with treating PAMM, the most appropriate treatment strategy remains 
controversial. Surgery as a primary treatment strategy for PAMM, ranges from abdominoperineal resection (APR) to wide local excision (WLE). However, previous studies comparing the difference in survival time of patients treated by APR or WLE identified no statistical difference $(10,13)$. In a number of studies, adjuvant chemotherapy (14), adjuvant radiation (15), neoadjuvant radiation (16) and immunotherapies (17) have also been used in PAMM, and certain curative effects were obtained. Preoperative CCRT has a proven role in the treatment of various malignant diseases, particularly in locally advanced rectal cancer (18). The predominant role of preoperative CCRT is to reduce locoregional and pelvic cavity recurrence, and to obtain a higher rate of sphincter preservation via tumor shrinkage. In addition, it facilitates the removal of potential micrometastasis and lessens distant metastases. In the present case, preoperative CCRT was performed in PAMM and the patient achieved a pathological partial response. Uner et al (16) reported a case of PAMM; the patient received radiation as the primary treatment rather than surgery and exhibited a similar outcome, indicating that neoadjuvant therapy may be beneficial to PAMM. The goal of therapy for PAMM should be to maximize the survival time as well as to improve quality of life. Preservation of sphincter function is, therefore, emphasized in surgery. Following CCRT, a decrease in tumor volume renders sphincter preservation feasible with negative margins. The timing of surgery following CCRT should also be considered. In the period following radiation therapy, tumor cells may be eradicated continually, and the adequate time between CCRT and surgery is 4-8 weeks (17).

KPS was a tool, developed as a subjective means of evaluating a patient's ability to perform ordinary tasks. It is a clinical prognosis index of the cancer patient population. In the current case, the patient exhibited a high KPS score following surgery, which indicates a good prognosis.

In conclusion, PAMM remains a rare disease that is associated with a poor prognosis. In order to improve the accuracy of diagnosis, physicians must be vigilant to the occurrence of PAMM, and immunohistochemistry should be a routine examination during colonoscopy pathology. The most effective treatment strategies for PAMM remain the focus of investigation. The therapeutic regimen of neoadjuvant CCRT together with sphincter-sparing surgery may guarantee a high quality of life and provide high efficacy, indicating that it may be an optimal strategy for patients with PAMM. Further studies are required to further evaluate the efficacy of this therapeutic regimen.

\section{References}

1. Wanebo HJ, Woodruff JM, Farr GH and Quan SH: Anorectal melanoma. Cancer 47: 1891-1900, 1981.

2. Pack GT and Oropeza R: A comparative study of melanoma and epidermoid carcinoma of the anal canal: A review of 20 melanomas and 29 epidermoid carcinomas (1930 to 1965). Dis Colon Rectum 10: 161-176, 1967.

3. Weston SD and Marren M: Malignant melanoma of the rectum. J Int Coll Surg 17: 403-416, 1952.

4. Balch CM, Gershenwald JE, Soong SJ, et al: Final version of 2009 AJCC melanoma staging and classification. J Clin Oncol 27: 61996206, 2009.

5. Goldman S, Glimelius B and Påhlman L: Anorectal malignant melanoma in Sweden. Report of 49 patients. Dis Colon Rectum 33: 874-877, 1990.

6. Moore W: Recurrent melanoma of the rectum after previous removal from the verge of the anus in a man aged sixty-five. Lancet 1: 290, 1857.

7. Yap LB and Neary P: A comparison of wide local excision with abdominoperineal resection in anorectal melanoma. Melanoma Res 14: 147-150, 2004.

8. Konstadoulakis MM, Ricaniadis N, Walsh D and Karakousis CP: Malignant melanoma of the anorectal region. J Surg Oncol 58: 118-120, 1995.

9. Zhang S, Gao F and Wan D: Effect of misdiagnosis on the prognosis of anorectal malignant melanoma. J Cancer Res Clin Oncol 136: 1401-1405, 2010.

10. Thibault C, Sagar P, Nivatvongs S, Ilstrup DM and Wolff BG: Anorectal melanoma - an incurable disease? Dis Colon Rectum 40: 661-668, 1997.

11. Zhong J,Zhou JN, Xu FP and Shang JQ: Diagnosis and treatment of anorectal malignant melanoma - a report of 22 cases with literature review. Ai Zheng 25: 619-624, 2006 (In Chinese).

12. Slingluff CL Jr, Vollmer RT and Seigler HF: Anorectal melanoma: clinical characteristics and results of surgical management in twenty-four patients. Surgery 107: 1-9, 1990.

13. Cooper PH, Mills SE and Allen MS Jr: Malignant melanoma of the anus: report of 12 patients and analysis of 255 additional cases. Dis Colon Rectum 25: 693-703, 1982.

14. Phade VR and Lawrence WR: Anorectal melanoma. Br J Surg 68: 667-668, 1981.

15. Ballo MT, Gershenwald JE, Zagars GK, et al: Sphincter-sparing local excision and adjuvant radiation for anal-rectal melanoma. J Clin Oncol 20: 4555-4558, 2002.

16. Uner A, Kilic D, Mentes BB, Egehan I and Dursun A: Neoadjuvant radiotherapy in anorectal malignant melanoma. Int J Clin Pract 57: 65-67, 2003.

17. Morton DL, Hsueh EC, Essner R, et al: Prolonged survival of patients receiving active immunotherapy with Canvaxin therapeutic polyvalent vaccine after complete resection of melanoma metastatic to regional lymph nodes. Ann Surg 236: 438-448, 2002.

18. Sanghera P, Wong DW, McConkey CC, Geh JI and Hartley A: Chemoradiotherapy for rectal cancer: an updated analysis of factors affecting pathological response. Clin Oncol (R Coll Radiol) 20: 176-183, 2008.

19. Bujko K, Nowacki MP and Liszka-Dalecki P: Radiation therapy for anorectal melanoma - a report of three cases. Acta Oncol 37: 497-499, 1998. 